\title{
OTIMIZAÇÃO DO PLANEJAMENTO DE ROTAS NA COLETA DE RESÍDUOS SÓLIDOS
}

\author{
T. P. B. VECCHI ${ }^{1}$, L. M. M. JORGE ${ }^{2}$, M. A. S. S. RAVAGNANI ${ }^{3}$ e P. R. PARAÍSO ${ }^{4}$ \\ ${ }^{1}$ Universidade Tecnológica Federal do Paraná, Departamento de Matemática \\ 2,3,4 Universidade Estadual de Maringá, Departamento de Engenharia Química \\ E-mail para contato: thelmapbv@gmail.com \\ lmmj@deq.uem.br \\ mauro.ravagnani@hotmail.com \\ paulo@deq.uem.br
}

\begin{abstract}
RESUMO - Neste trabalho apresenta-se um modelo de otimização para a solução do problema de planejamento de coleta e transporte de resíduos sólidos em cidades de médio porte. Como resultados finais espera-se promover redução de custos aos cofres públicos, bem como benefícios ao meio ambiente. O modelo matemático desenvolvido é formulado como um problema de Programação Linear Mista com variáveis inteiras (PLMI) e transcrito para a linguagem algébrica GAMS. A aplicação prática foi realizada com dados coletados na região central de uma cidade brasileira com aproximadamente 90.000 habitantes. O modelo determinístico utilizado permitiu que uma solução ótima fosse encontrada após a inclusão de restrições que eliminaram o aparecimento de sub-rotas. Concluiu-se que as rotas otimizadas permitem uma redução de $20 \%$ na distância total percorrida, o que pode gerar uma economia de $\mathrm{R} \$ 300,00 /$ dia referente a gastos de manutenção e combustível dos caminhões.
\end{abstract}

\section{INTRODUÇÃO}

A urbanização se intensificou no Brasil a partir da década de 70. Atualmente, segundo o IBGE, 84\% dos brasileiros residem nos centros urbanos. Concomitantemente à urbanização, cresceu também a produção de resíduos domésticos que necessitam ser coletados e dispostos em locais adequados.

A coleta dos resíduos é uma tarefa dispendiosa para a maioria dos municípios brasileiros. Um gerenciamento eficiente, que reduza os custos operacionais e o impacto ambiental com a queima de combustível no transporte destes materiais, se faz necessário. Segundo Cunha e Caixeta (2002), os municípios gastam grandes quantias do seu orçamento (7\% a 15\%) com o gerenciamento do lixo. Estima-se que 50\% desse montante é gasto com o transporte e a coleta do mesmo. Dessa forma, esse trabalho tem como objetivo a otimização de rotas da coleta de resíduos na região central da cidade de Campo Mourão-PR, a fim de que se possa contribuir com uma redução de custos aos cofres públicos, bem como proporcionar benefícios ao meio ambiente.

Vários trabalhos foram desenvolvidos com o intuito de otimizar rotas para a coleta de resíduos domésticos. Alguns utilizam modelos exatos e outros, heurísticas, devido ao grande 
número de variáveis e restrições do problema. A seguir, são relatadas algumas pesquisas realizadas nessa área, em âmbito nacional e internacional.

Paes (2004) propôs uma otimização de rotas para a coleta de resíduos domésticos, utilizando a metaheurística Grasp. Godinho e Junqueira (2006) estudaram aplicações do Problema do Carteiro Chinês (PCC) para a coleta de resíduos domésticos na cidade de São Carlos/SP. Souza e Rangel (2009) utilizaram uma adaptação do algoritmo do Problema do Carteiro Chinês para otimizar a rota de coleta de resíduos sólidos em um bairro de Volta Redonda/RJ. Rigonatti et al. (2012), realizaram um estudo de caso numa região da cidade de São Paulo, utilizando modelos do Problema do Carteiro Chinês Misto para otimizar a rota dos caminhões de coleta de resíduos. WY et al. (2013), propuseram um algoritmo iterativo, o "the rollon-rolloff vehicle routing problem with time windows" (RR-VRPTW), de abordagem heurística, para otimizar rotas de coleta de resíduos.

Agha (2006) utilizou de Programação Linear Inteira Mista ao otimizar rotas para a coleta de resíduos de uma cidade de 44 mil habitantes, situada na Faixa de Gaza, utilizando um modelo de Problema de Roteamento de Veículos (PRV). Seus resultados favorecem um decréscimo de $24 \%$ na distância percorrida pelos caminhões e uma economia para o município de US\$1140,00/ mês.

Como se observa acima, uma variedade de pesquisas já foi desenvolvida nessa área. Porém, a realizada por Agha (2006), demonstrou ser mais viável por considerar variáveis e restrições coerentes com o problema em questão, o que justifica ter sido escolhida como base para este trabalho. O modelo matemático utilizado pelo autor é um problema de Programação Linear Mista com variáveis inteiras (PLMI), denominado Problema de Roteamento de Veículos (PRV).

Dantzig e Ramser introduziram o PRV em 1959 ao considerar uma frota ilimitada de veículos idênticos, estacionados numa garagem, e um conjunto de clientes com procura conhecida. Pretendiam encontrar um conjunto de rotas que permitisse satisfazer a procura dos clientes com custo total mínimo. As rotas tinham início e fim na garagem e deveriam satisfazer a seguinte condição: a soma das procuras dos clientes visitados não deveria ultrapassar a capacidade do veículo. Os custos foram identificados como as distâncias físicas percorridas (GODINHO, 2011).

Esses problemas variam quanto a sua complexidade, dependendo do número de variáveis e restrições considerado em sua formulação. Alguns podem ser classificados como intratáveis conforme seu tamanho. Mesmo utilizando computadores de última geração, dificuldades podem ser encontradas, pois estas residem na natureza combinatória desse tipo de problema. Eles são considerados NP-Hard e, em situações complexas, pode-se utilizar técnicas para alcançar soluções próximas da ótima, como as heurísticas (MIURA, 2003).

O modelo matemático proposto por Agha (2006) demonstrou ser robusto para a aplicação com dados reais, porém não evitou o surgimento de sub-rotas na solução (rotas não viáveis devido ao fato de não iniciarem na garagem dos caminhões e terminarem no aterro sanitário). Desta forma, esse trabalho apresenta sua contribuição ao propor algumas alterações 
no modelo proposto por Agha (2006), incluindo restrições que eliminam sub-rotas da solução, tornando-a assim compatível com a esperada para o problema.

\section{MATERIAIS E MÉTODOS}

\subsection{Materiais}

A coleta dos resíduos sólidos na cidade de Campo Mourão, noroeste do Paraná, é realizada por uma empresa terceirizada que presta serviços de coleta indiferenciada e de coleta seletiva. Para a realização desta pesquisa, foram consideradas as rotas dos caminhões de coleta indiferenciada na região central da cidade. Este trabalho é realizado de segunda-feira a sábado, durante o período da noite e, para isso, a empresa utiliza de dois caminhões compactadores homogêneos (cada um com capacidade aproximada de 17 toneladas).

\subsection{Métodos}

No presente trabalho um modelo matemático baseado no trabalho de Agha (2006) foi desenvolvido, com a diferença de que as possíveis sub-rotas na solução sejam evitadas a partir do uso de uma restrição proposta por Ahuja et al. (1993). As alterações propostas ao modelo de Agha (2006) aparecem em várias equações, ao considerar situações de desigualdade $(<$, $\neq$ ), entre os índices $i, j, t$. Fato que elimina viagens não permitidas (de um nó para ele mesmo) e reduz significativamente o número de equações geradas pelo modelo.

O modelo de Agha (2006) é um Problema de Roteamento de Veículos (Problema de Programação Linear Mista com variáveis inteiras - PLMI) implementado para otimizar as rotas dos caminhões de coleta de resíduos em Deir El-Balah, na Faixa de Gaza. O modelo considera as seguintes variáveis e parâmetros:

$$
\begin{aligned}
& x_{i j k}=\left\{\begin{array}{l}
1, \text { se o carro } k \text { viaja de } P_{i} \text { a } P_{j}, \\
0, \text { caso contrário. }
\end{array}\right. \\
& z_{i k}=\left\{\begin{array}{l}
1, \text { se o carro } k \text { recolhe containers em } P_{i}, \\
0, \text { caso contrário. }
\end{array}\right. \\
& y_{i k}=\text { número de containers em } P_{i} \text { recolhidos pelo carro } k \\
& d_{i j}=\text { distância da viagem de } P_{i} \text { a } P_{j} \\
& f_{i}=\text { número de containers a serem recolhidos em } P_{i} \\
& c_{k}=\text { capacidade do veículo } k \text { em toneladas. }
\end{aligned}
$$

Os dados necessários para a implementação do modelo matemático desenvolvido na região central da cidade de Campo Mourão-PR, são: um conjunto de nós, $\operatorname{Nos}(i)=\{1,2, \ldots$, $71\}$; um conjunto que representa os caminhões destinados à coleta do lixo, $k=\{1,2\}$; um 
parâmetro associado à capacidade dos caminhões, $c_{k}$; um parâmetro associado à quantidade de resíduos em cada ponto de coleta, $f_{i}$; uma tabela de distâncias, de ordem 71 x $71, d(i, j)$.

Os caminhões partem da garagem da empresa, coletam os resíduos na região e finalizam a rota no aterro sanitário. Neste trabalho representou-se a garagem pelo nó 1 , os pontos de coleta pelos nós de números 2 a 70 (localizados nas esquinas da região) e o aterro sanitário pelo nó 71. A quantidade de resíduos sólidos coletados diariamente, em média, nessa região é de 33 toneladas (dados fornecidos pela empresa que realiza a coleta na cidade).

O modelo matemático, com algumas alterações, é o seguinte:

$$
\operatorname{Min} \sum_{k=1}^{2} \sum_{i=1}^{70} \sum_{j=2}^{71} d_{i j} x_{i j k}, \text { para todo } i \neq j
$$

$\sum_{i=2}^{70} y_{i k} \leq c_{k}, \quad k=1,2$

$\sum_{k=1}^{2} y_{i k}=f_{i}, \quad i=2, \ldots, 70$

$\sum_{i=1}^{70} x_{i t k}=\sum_{j=2}^{71} x_{t j k}, \quad t=2, \ldots, 70 ; k=1,2 ; i \neq t ; j \neq t$

$\sum_{i=2}^{70} x_{i(71) k}=\sum_{j=2}^{70} x_{(1) j k}=1, k=1,2$

$\sum_{j=2}^{71} x_{i j k} \geq z_{i k}, \quad i=2, \ldots, 70 ; k=1,2 ; i \neq j$

$y_{i k} \leq f_{i} z_{i k}, \quad i=2, \ldots, 70 ; k=1,2$

$x_{i j k}+x_{j i k} \leq 1, i, j=2, \ldots, 70 ; i<j ; k=1,2$

$\sum_{j=2}^{71} x_{i j k} \leq 1, i=1,2, \ldots, 70 ; i \neq j ; k=1,2$

$x_{i j k}=0$ ou $1, \quad z_{i k}=0$ ou $1, \quad y_{i k}=$ inteiro positivo

Segundo Agha (2006), a restrição (2) garante que a capacidade dos veículos seja considerada; a restrição (3) garante que os containers serão recolhidos; as restrições (4) e (5) 
estão relacionadas à continuidade do fluxo; se um veículo pega containers em um determinado ponto de coleta, ele deve visitar este ponto e isso está representado nas restrições de número (6) e (7); a restrição (8) garante que o container será recolhido por apenas um veículo; a restrição (9) é acrescentada para fazer com que o veículo se mova de um ponto $i$ para apenas um ponto $j$ e vice-versa. A restrição (10) refere-se à integralidade das variáveis. $\mathrm{O}$ autor não considerou em seu modelo as restrições que eliminam possíveis sub-rotas geradas ao implementá-lo, porém mencionou a necessidade de incluí-las, caso necessário.

A restrição da Equação 8 no modelo de Agha $\left(x_{i j k}+x_{j i k} \leq 1, i, j=1,2, \ldots n ; k=\right.$ $1,2, \ldots, k)$ impõe que não ocorra um trajeto de ida e outro de volta entre dois nós do problema. Ao que parece, o autor sugeriu essa restrição para garantir que o container fosse recolhido por apenas um veículo, equivocadamente. A fim de suprir essa falha, incluiu-se a seguinte restrição ao modelo:

$$
\sum_{k=1}^{2} z_{i k}=1, \quad i=2, \ldots, 70
$$

A restrição da Equação 11 considera que a variável $z_{i k}$ seja 1 para um único veículo. Por exemplo, a equação $z_{51}+z_{52}=1$ faz com que os resíduos no nó 5 sejam recolhidos por apenas um caminhão. Se $z_{51}=1, z_{52}=0$, todo o resíduo será recolhido pelo caminhão 1 .

Além desta, outra restrição é proposta para o modelo:

$$
\sum_{k=1}^{2} x_{i j k} \leq 1, \quad i, j=2, \ldots, 70 ; i \neq j
$$

A restrição da Equação 12 impede que um mesmo trajeto entre os nós $i$ e $j$ seja realizado por mais de um caminhão. Por exemplo, a inequação $x_{341}+x_{342} \leq 1$ garante que o trajeto do nó 3 para o nó 4 seja realizado pelo caminhão 1 ou pelo caminhão 2 (não por ambos), ou ainda que esse trajeto não seja realizado por nenhum dos dois caminhões.

As restrições correspondentes às Equações 11 e 12 foram incluídas no modelo para a otimização de rotas da coleta de resíduos na região central de Campo Mourão. O modelo descrito foi implementado na linguagem algébrica GAMS, versão 22.6, resolvido a partir do solver Cplex, versão 11.0, em um computador com processador intel core i5 e 4 GB de memória RAM. Uma solução ótima foi obtida, porém sub-rotas foram detectadas.

O conjunto de restrições do modelo de PRV, proposto por Ahuja et al. (1993), foi considerado para a eliminação das sub-rotas. Segundo o autor, tem-se:

$$
\sum_{i \in Q} \sum_{j \in Q} y_{i j} \leq|Q|-1 \text {, para todo subconjunto } Q \text { de }\{2,3, \ldots, n\}
$$


O conjunto Q representa todo subconjunto de nós que podem formar sub-rotas. Como exemplo, a Figura 1 representa, hipoteticamente, a situação em questão. A rota deveria começar no nó 1 e terminar no nó 71, passando por todos os demais nós. Porém uma sub-rota aparece, formada pelos nós 2, 3 e 4, a qual é mostrada na Figura 1.

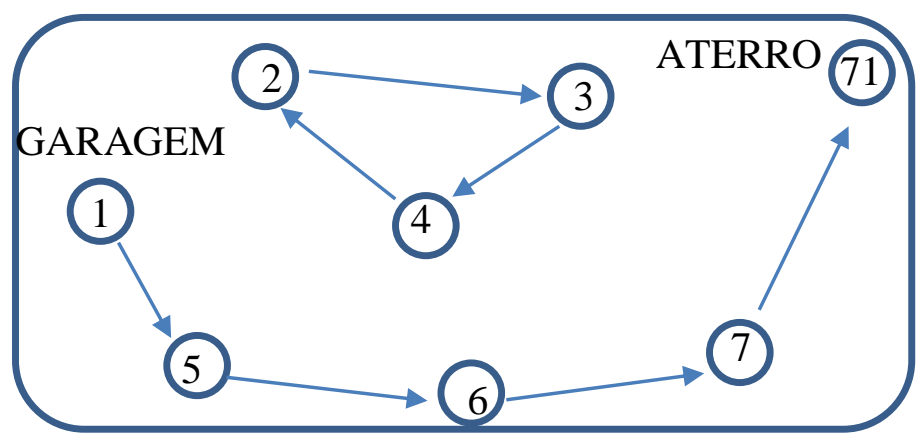

Figura 1 - Exemplo de sub-rota gerada pelos nós 2, 3 e 4.

Entretanto, considerar todas as sub-rotas possíveis para o problema em questão (71 nós) torna-se inviável computacionalmente, pois o número de combinações de nós é da ordem de $2^{69}$ (a garagem e o aterro são excluídos deste cálculo). Ao observar as sub-rotas obtidas nas primeiras iterações do modelo, pôde-se perceber que a maioria delas continham 3, 4 ou 5 nós. Então, desenvolveu-se um programa em visual basic para formar todas as combinações possíveis de 3, 4 e 5 nós que atendiam às restrições de distâncias entre eles (nós próximos entre si geralmente formam as sub-rotas), evitando assim a formação de combinações desnecessárias.

\section{RESULTADOS}

Após acrescentar as restrições que eliminam as sub-rotas de 3, 4 e 5 nós, outras subrotas apareceram na solução, com quantidades maiores de nós. Assim, ao término de cada iteração do modelo, as sub-rotas surgidas foram eliminadas (utilizando a Equação 13) e após algumas iterações, uma solução ótima foi encontrada.

A solução é composta de duas rotas ótimas, uma para cada caminhão, cada uma com início na garagem (nó 1) e término no aterro sanitário (nó 71), que encontra-se a $12 \mathrm{~km}$ de distância do nó de número 25, numa estrada vicinal (devido a escala, este nó não aparece no mapa das Figuras 2 e 3). Todos os pontos de coleta foram visitados e todo resíduo coletado (por um único caminhão em cada ponto de coleta), não extrapolando a capacidade dos caminhões.

Nas Figuras 2 e 3 podem-se observar as rotas geradas para cada um dos dois caminhões, na região central da cidade: 


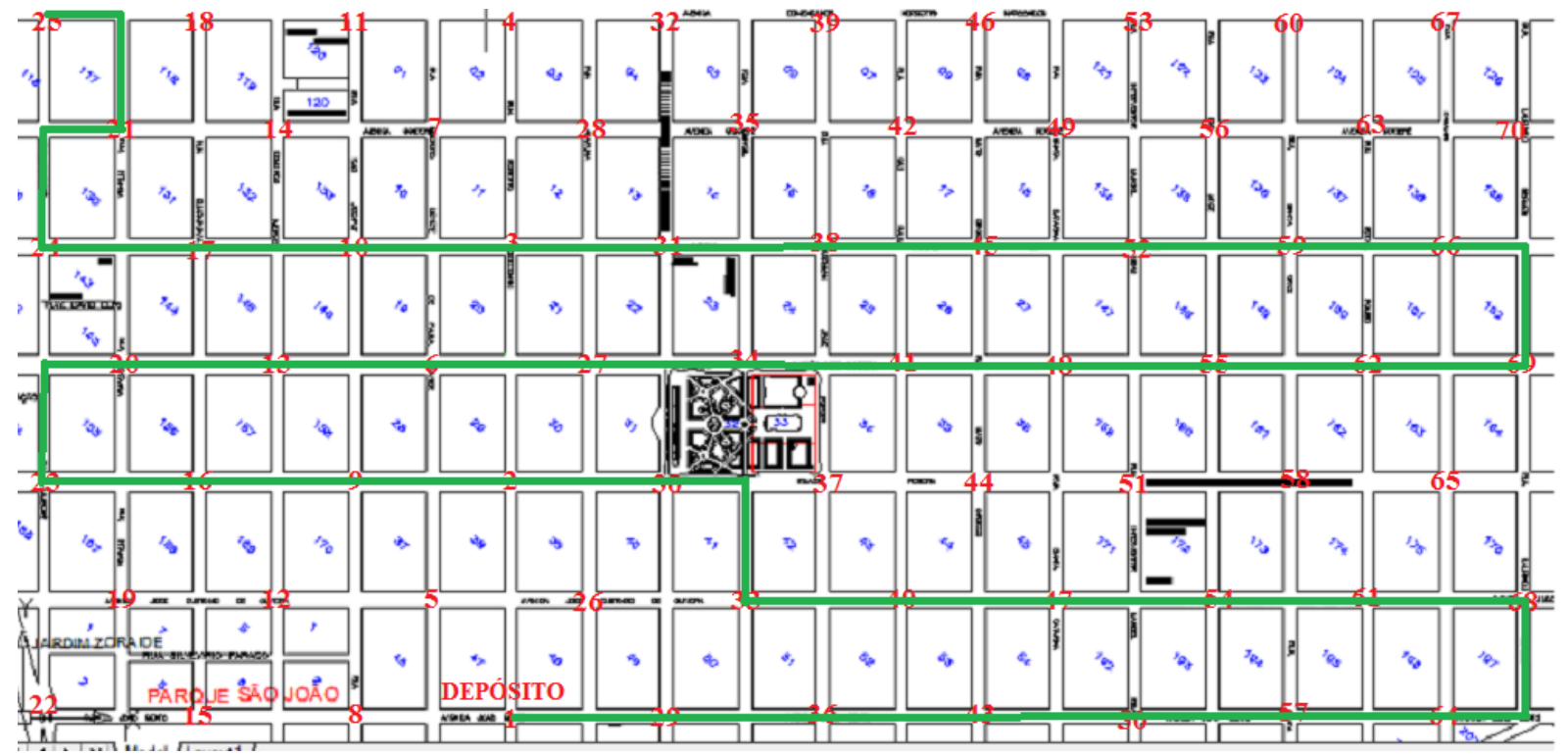

Figura 2 - Rota gerada para o caminhão 1 (Planta da região em AUTOCAD 2007)

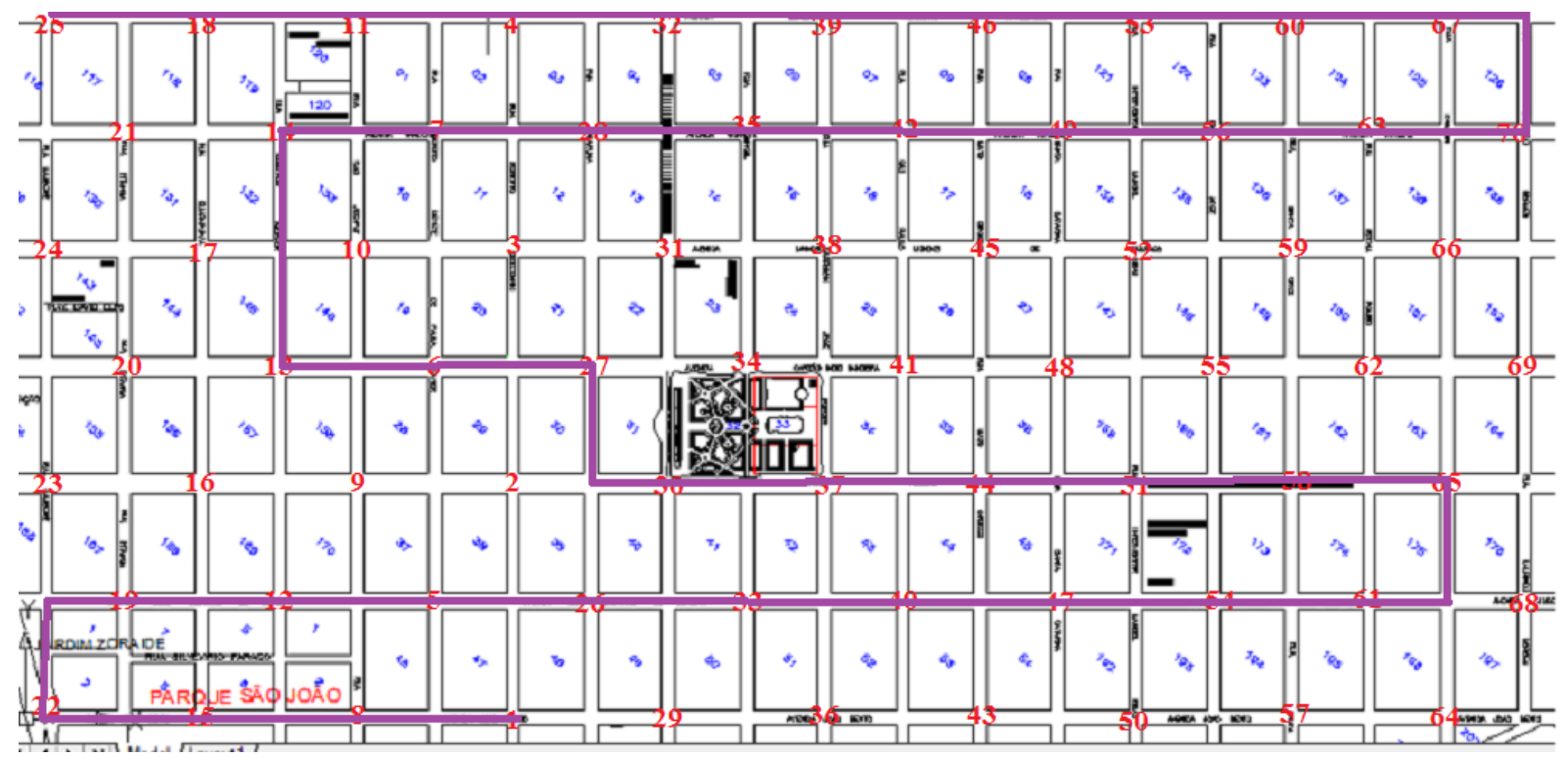

Figura 3 - Rota gerada para o caminhão 2 (Planta da região em AUTOCAD 2007)

Segundo a empresa que realiza o trabalho de coleta de resíduos na cidade de Campo Mourão, se gasta em média $\mathrm{R} \$ 25,00 / \mathrm{km}$ rodado, referente a gastos com manutenção e combustível dos caminhões. Sendo assim, as rotas otimizadas, quando comparadas com as realizadas pela empresa coletora, permitem uma economia de $\mathrm{R} \$ 300,00 /$ dia, considerando algumas simplificações que precisaram ser feitas para que o modelo pudesse ser utilizado. Essas simplificações ocorreram principalmente na quantidade de nós considerados, pois limitações dessa grandeza ocorrem quando se trata de Problema de Roteamento de Veículos resolvido a partir de modelos determinísticos. A princípio, todas as esquinas da região central da cidade foram consideradas como pontos de coleta, totalizando 141 nós. Mas, devido ao 
grande número de combinações entre os nós, as restrições que evitam sub-rotas na solução não obtiveram sucesso. Sendo assim, reduziu-se essa quantidade para 71 nós (uma esquina sim e outra não) e esse problema pôde ser evitado.

\section{CONCLUSÕES}

O modelo desenvolvido, baseado no modelo de Agha (2006), demonstrou ser robusto para a determinação de rotas otimizadas para os caminhões de coleta de resíduos sólidos. $\mathrm{O}$ resultado obtido é proveniente de um modelo exato, cuja limitação está na quantidade de nós considerados como pontos de coleta. Por isso, simplificações desta grandeza foram necessárias para que o mesmo pudesse ser utilizado com sucesso.

As rotas otimizadas ao serem comparadas com as realizadas pela empresa coletora, proporcionam uma redução na distância total percorrida pelos caminhões de $20 \%$, o que pode acarretar uma economia de $\mathrm{R} \$ 300,00 /$ dia.

Sendo assim, os resultados obtidos a partir desta pesquisa se colocados em prática, podem proporcionar economia aos cofres públicos, bem como benefícios ao meio ambiente, situações essas importantes para o bem estar da população.

\section{REFERENCIAS}

AGHA, S. R. Optimizing Routing of Municipal Solid Waste Collection Vehicles in Deir El-Balah Gaza Strip. The Islamic University Journal, 2006.

AHUJA, R.K.; MAGNANTI, T.L.; ORLIN, J.B. Network Flows: Theory, Algorithms, and Applications. Englewood Cliffs, NJ, Prentice Hall, 1993.

CUNHA, V.; CAIXETA, F. J. V. Gerenciamento da coleta de Resíduos Sólidos Urbanos: Estruturação e Aplicação de Modelo Não-Linear de Programação por Metas. Gestão e Produção, 2002.

GODINHO, M. T. Modelos Envolvendo Variáveis Com Dependências Temporais para Problemas de Roteamento. Tese de Doutorado. Universidade de Lisboa, 2011.

GODINHO, M.; JUNQUEIRA, R. A. R. Problema do Carteiro Chinês (CPP): Escolha de Métodos de Solução e Análise de Tempos Computacionais. Produção, v. 16, 2006.

IBGE: Censo 2010. Disponível em: http://censo2010.ibge.gov.br/noticias-censo? $\mathrm{id}=3 \&$ idnoticia=1766\&t=censo-2010-populacao-brasil-190-732-694-pessoas \& view=noticia . Acesso em 20/02/2014.

MIURA, M. Resolução de um Problema de Roteamento de Veículos em uma Empresa Transportadora. Escola Politécnica da Universidade de São Paulo, 2003.

PAES, F. G. Um Tratamento GRASP Híbrido do PCCM Destinado a Otimização de Rotas. XXVIII CNMAC Congresso Nacional de Matemática Computacional, v. 1. p. 1-7, São Paulo, 2005.

RIGONATTI, A.; RODRIGUES, J. A. V.; BATISTA, P. L. F.; GOMES, M. J. N. Experiências com coleta de lixo domiciliar e aplicação de modelos do problema do carteiro chinês misto: estudo de caso no Jardim Europa. Engenharia, 2012.

SOUSA, B. C. S.; RANGEL, L. A. D. Determinação de Rota Ótima de um Caminhão de Coleta de Resíduos por um Bairro Baseado no Problema do Carteiro Chinês. VIII SEGET - Simpósio de Excelência em Gestão e Tecnologia, Resende, 2009.

WY J., KIM B., KIM S. The rollon-rolloff waste collection vehicle routing problem with time windows (RR-VRPTW), European Journal of Operational Research, 2013. 